\title{
Arritmia cardiaca posterior a anestesia espinal en cesárea
}

\author{
Cardiac arrhythmia after to spinal anesthesia in cesárean
}

Patricia Alegre Andrade ${ }^{1}$

\begin{abstract}
La arritmia y taquicardia son muy comunes en las embarazadas, aunque el bloqueo espinal pueda ser una técnica anestésica segura, la taquicardia grave, la parada cardiaca y otras arritmias son relatadas durante las prácticas de raquianestesia. La bupivacaina y levobupivacaina pueden aumentar el intervalo PR y la duración del QRS y prolongar la conducción cardiaca. La dexmedetomidina tiene propiedades simpaticolíticas, sedativas, estabilidad respiratoria sin depresión ventilatoria, amnésicas y analgésicas, los efectos adversos de la dexmedetomidina son la hipertensión inicial, hipotensión, naúseas, bradicardia, fibrilación atrial, edema pulmonar, oliguria y sed. Paciente de 33 años de edad con embarazo de 39,2 sem. Sin antecedentes patológicos. Recibe anestesia espinal y a los 10 min bradicardica de 39 corregida con atropina y posterior con arritmia sinusal. En conclusión la presencia de arritmias cardiacas puede deberse a varios factores, es trascendental la vigilancia y monitoreo continuo del electrocardiograma para reconocer y corregir de manera oportuna.

Palabras claves: arritmia sinusal, dexmedetomidina, anestesia espinal

\section{Resumen}

Arrhythmia and tachycardia are very common in pregnant women, although spinal block may be a safe anesthetic technique, severe tachycardia, cardiac arrest and other arrhythmias are reported during spinal anesthesia practices. Bupivacaine and levobupivacaine can increase the PR interval and the duration of QRS and prolong cardiac conduction. Dexmedetomidine with sympatholytic, sedative, respiratory stability without ventilatory depression, amnesic and analgesic properties, the adverse effects of dexmedetomidine are initial hypertension, hypotension, nausea, bradycardia, atrial fibrillation, pulmonary edema, oliguria and thirst. 33-year-old patient with a pregnancy of 39.2 weeks. No pathological history she received spinal anesthesia and at 10 min bradycardia of 39 corrected with atropine and later with sinus arrhythmia. In conclusion, the presence of cardiac arrhythmias can be due to several factors, the continuous monitoring and monitoring of the electrocardiogram to recognize and correct in a timely manner is transcendental.

Keywords: sinus arrhythmia, dexmedetomidine, spinal anesthesia
\end{abstract}

$\mathrm{L}$ as arritmias cardíacas son causa importante de morbilidad y mortalidad durante el período perioperatorio, así como en pacientes críticos en unidades de cuidados intensivos (UCI) ${ }^{1}$.

Los agentes anestésicos pueden presentar actividad proarritmica y antiarritmica al inducir actividad eléctrica por medio de varios mecanismos ${ }^{2}$.

La anestesia neuroaxial es considerada como método preferido de la analgesia y anestesia obstétrica para la atención de parto vaginal o cesárea. Se evita los efectos de las drogas al feto, los anestesiólogos generalmente no usan sedantes y otras drogas antes del nacimiento ${ }^{3}$.

La arritmia y la taquicardia son muy comunes en las embarazadas, aunque el bloqueo espinal pueda ser una técnica anestésica segura, la taquicardia grave, la parada cardiaca y otras arritmias son relatadas durante las prácticas de raquianestesia ${ }^{4}$.

La bupivacaina y levobupivacaina pueden aumentar el intervalo PR y la duración del QRS de este modo, prolongar la conducción cardiaca ${ }^{5}$.

La dexmedetomidina es un potente y altamente selectivo un alto seleccionador agonista de los adrenoceptores $\alpha-2$ con

${ }^{1}$ Médico Anestesiólogo - Hospital Obrero N ${ }^{\circ} 2$ Caja Nacional de Salud, Cochabamba , Bolivia

${ }^{*}$ Correspondencia a: Patricia Alegre Andrade

Correo electrónico: wonderful122.paa@gmail.com

Recibido el 07 de octubre de 2019. Aceptado el 20 de marzo de 2020 propiedades simpaticolíticas, sedativas, estabilidad respiratoria sin depresión ventilatoria, amnésicas y analgésicas, que ya ha sido descrito como un suplemento útil y seguro en varias aplicaciones clínicas ${ }^{6}$.

Los efectos adversos de la dexmedetomidina son la hipertensión inicial, hipotensión, náuseas, bradicardia, fibrilación atrial, edema pulmonar, oliguria y $\operatorname{sed}^{7}$. La sobredosis puede causar bloqueo atrioventricular de primero o segundo grado. La mayor parte de los efectos adversos asociados al uso de la dexmedetomidina, ocurre durante o inmediatamente después de la dosis de ataque ${ }^{8}$.

\section{Presentación del caso}

Paciente femenina de 33 años de edad, procedente de Cercado - Cochabamba, en fecha 03 de junio de 2019 acude al servicio de emergencias de Gineco-Obstetricia del Hospital Obrero N ${ }^{\circ}$ "Caja Nacional de Salud".

Paciente cursa con embarazo de 39, 2 semanas de gestación por último periodo menstrual (FUM), Cesárea previa, formula obstétrica: G2C1P0A0. Con leve dolor abdominal.

El servicio de Gineco-obstetricia decide interrumpir el embarazo con los diagnostico de embarazo de 39,2 semanas, cesárea previa y pródromos de trabajo de parto, por lo cual nos solicitan valoración por anestesiología. Paciente refiere ayuno a líquido claros mayor a dos horas y a continuación se detallan la valoración pre-anestésica: 


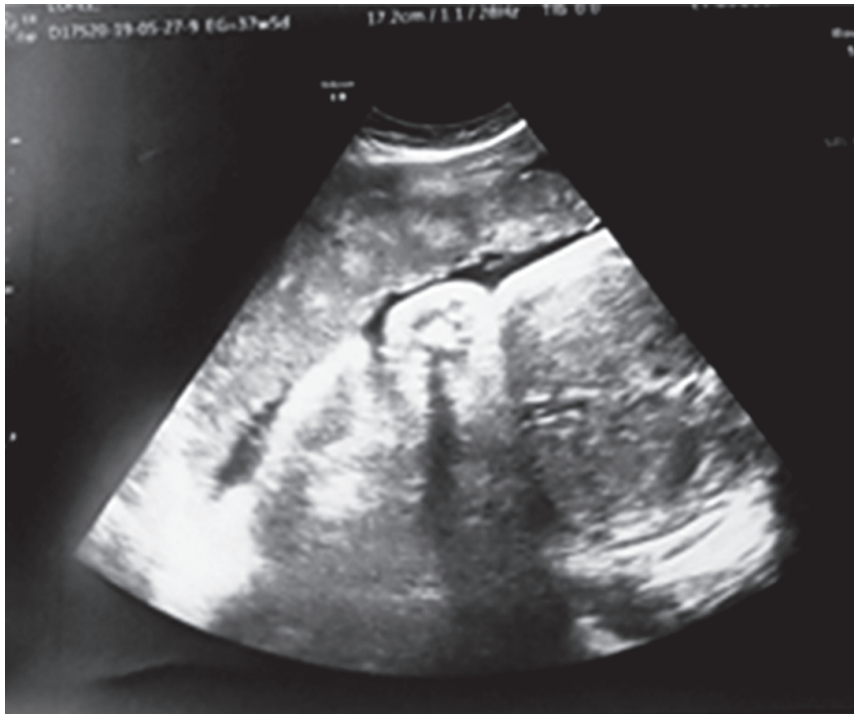

Figura 1. Placenta anterior, no previa. Grado I.

\section{Antecedentes personales}

- No refiere antecedentes patológicos de relevancia tampoco refiere alergias.

- Hábitos personales: no refiere alcoholismo, tabaquismo.

- Antecedentes quirúrgicos: amigdalectomia bilateral hace 22 años bajo anestesia general sin complicaciones, cesárea hace 6 años por circular de cordón con anestesia espinal sin complicaciones.
- Antecedentes Gineco-obstetricos: G2 C1 P0 A0.

- Medicamentos: sulfato ferroso 200 mg, 1 comprimido vía oral cada día.

\section{Examen Físico General:}

Paciente con ansiedad, afebril, con mucosas húmedas y rosadas, hemodinámicamente estable.

Signos vitales: presión arterial de 110/70 mmhg. Frecuencia cardiaca de 80 latidos por minuto. Saturación de oxígeno a medio ambiente $96 \%$.

\section{Examen físico regional:}

- Vía Aérea: dientes incompletos, Mallampati I, Distancia Tiro - Mentoneana mayor a $6 \mathrm{~cm}$. Distancia esterno-mentoneana mayor a $10 \mathrm{cms}$.

- Cuello: móvil, simétrico.

- Neurológico: conciente, orientado. Glasgow:15/15.

- Corazón: rítmico, regular.

- Pulmones: murmullo vesicular conservado.

- Abdomen: globoso a expensas de útero grávido, con altura uterina de $29 \mathrm{~cm}$. Producto único, en situación longitudinal, presentación cefálica, dorso izquierdo, frecuencia cardiaca fetal de 150 latidos por minuto y movimientos fetales activos.

- Extremidades: normotróficas, normotónicas con

Tabla 1. Laboratorios de la paciente

\begin{tabular}{|c|c|c|c|}
\hline & \multicolumn{2}{|c|}{ Laboratorio de la paciente } & \multirow{2}{*}{$\begin{array}{l}\text { Valor normal } \\
4,20-5,80\end{array}$} \\
\hline \multirow{5}{*}{ Hemograma } & Glóbulos rojos* & 4590000 & \\
\hline & Hemoglobina & $12,2 \mathrm{~g} / \mathrm{dl}$ & $11-16,5 \mathrm{~g} / \mathrm{dl}$ \\
\hline & Hematocrito & $36,5 \%$ & $35-54 \%$ \\
\hline & Glóbulos blancos & $7750 \mu \mathrm{L}$ & $4,5-10,0 \mu \mathrm{L}$ \\
\hline & Plaquetas ${ }^{\circledR}$ & 278000 & $150-400$ \\
\hline \multirow[t]{2}{*}{ Química sanguínea } & Glicemia & $60 \mathrm{mg} / \mathrm{dl}$ & $70-110 \mathrm{mg} / \mathrm{dl}$ \\
\hline & Creatinina & $0.8 \mathrm{mg} / \mathrm{dl}$ & $0,5-1,5 \mathrm{mg} / \mathrm{dl}$ \\
\hline \multirow{4}{*}{ Otros } & TP/INR & $10,1 / 1$ & $10 \mathrm{seg} / 1.0$ \\
\hline & Grupo Sanguíneo & $\mathrm{O}(+)$ & \\
\hline & Prueba de VIH & No reactivó & \\
\hline & Prueba de Hepatitis B-C & Negativo & \\
\hline
\end{tabular}

* Glóbulos rojos: $(\mathrm{UL}) \quad$ • Plaquetas: $(\mu \mathrm{L})$ 


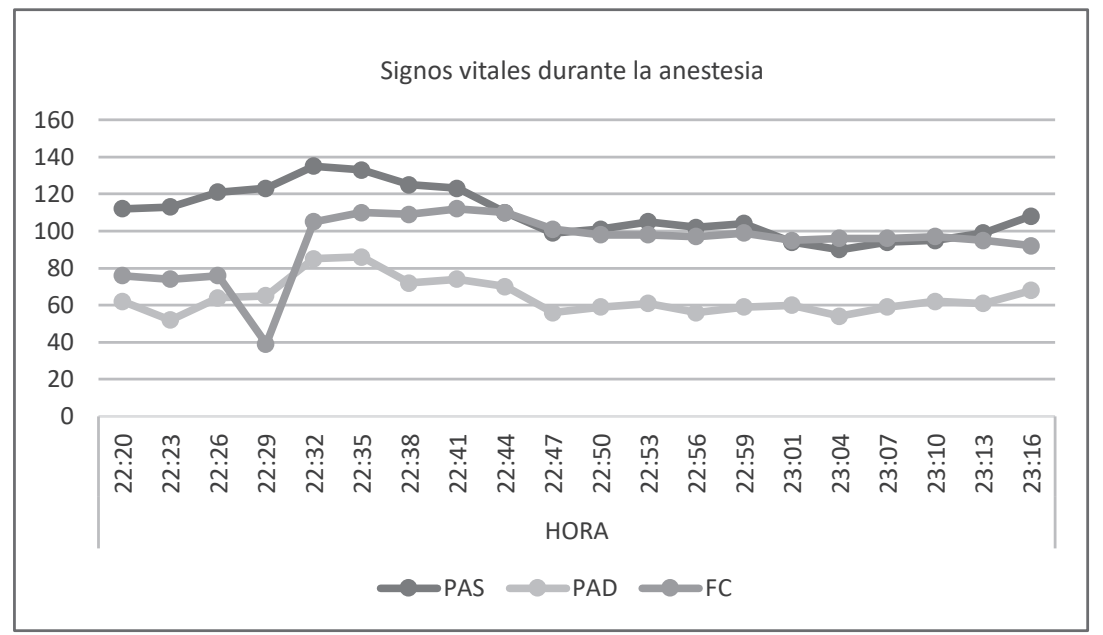

Figura 2. Registro de presión arterial sistólica, presión arterial diastólica y frecuencia cardiaca.

pulsos periféricos palpables, con llenado capilar menor a 2 segundos.

\section{Examen complementario}

- Hemograma y química sanguínea (Tabla 1)

- Ecografía Obstétrica: placenta anterior, no previa. Grado I. (Figura 1)

- No cuenta con Electrocardiograma de inicio por protocolo del servicio.

\section{Impresión Diagnostica:}

- Embarazo de 39,2 semanas

- Cesárea previa

- Pródromos de trabajo de parto

- Feto único vivo
- Clasificación de estado físico de la Sociedad Americana de Anestesiología ASA II

Plan Anestésico: anestesia neuroaxial, analgesia posquirúrgica, prevención de hipotensión, náuseas y vómitos.

Paciente en quirófano se efectúo la monitorización básica de signos vitales, encontrándose con PA: 112/62 mmhg, FC:76x', SatO2: 96\%. Paciente en sedestación se realizó asepsia y antisepsia, punción lumbar por abordaje mediano con aguja raquídea punta de lápiz Whitacre $\mathrm{N}^{\circ} 27$ a nivel del espacio L4 -L5; confirmando la correcta colocación de la aguja por el libre flujo de líquido cefalorraquídeo, se administró mezcla anestésica que contiene: $10 \mathrm{mg}$ de Bupivacaína hiperbárica al 0,5\% y dexmedetomidina $3 \mu \mathrm{g}$. Paciente en posición supina con la cuña pélvica para el desplazamiento del útero hacia la izquierda. Se efectuó la monitorización

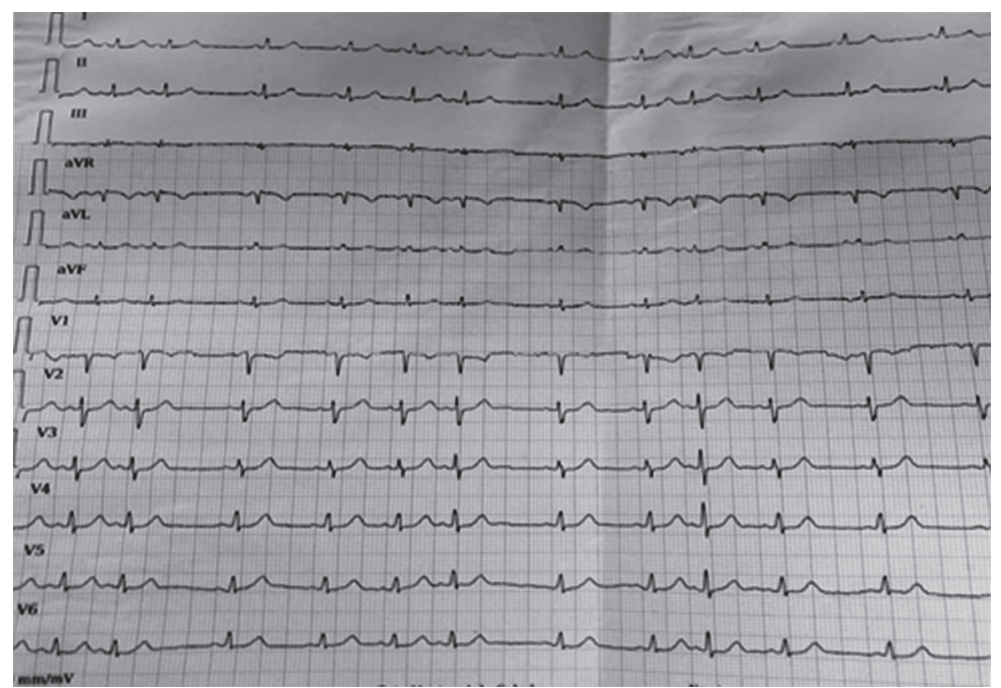

Figura 3. Electrocardiograma de la paciente. 
básica de presión arterial no invasiva, electrocardiograma continuo, frecuencia cardiaca y oximetría de pulso; se realizó control de estos parámetros cada 3 minutos transquirúrgico. Nivel de dermatoma alcanzado T5. A los 10 minutos de la anestesia se observa aumento de la presión arterial media en $6 \mathrm{mmHg}$ de la basal y bradicardia de 39 latidos por minuto, se administra atropina $0,5 \mathrm{mg}$ IV, y PA 100/58 mmg. Con buen resultado de 105 latidos por minuto y se mantiene durante todo el procedimiento anestésico entre 108 - 95 latidos por minuto (Figura 2). Se administra soluciones cristaloides a razón de $15 \mathrm{ml} / \mathrm{kg}$, se obtiene recién nacido de sexo femenino con APGAR de 8 al minuto y APGAR 9 a los 5 minutos, posteriormente se administra oxitocina 3 UI endovenosos en bolo en tres minutos.

Nuestra paciente presento un aumento de la presión arterial dentro los 10 primeros minutos

Se evidencia alteraciones en el electrocardiograma sin repercusiones en la presión arterial (Figura 3). Al concluir se solicita valoración por cardiológica.

El servicio de cardiología menciona que es un arritmia sinusal transitoria que solo amerita control, sin medicamentos.

La paciente es dada de alta a los dos días postquirúrgico sin ningún problema cardiaco, con ritmo sinusal y su control por obstetricia en 10 días.

\section{Discusión}

La presentación del caso clínico es importante para determinar las causas de bradicardia, especialmente en anestesia obstétrica.

La anestesia regional es la técnica preferida para la cesárea porque permite el contacto materno - fetal, sin manipulación de la vía aérea y mantención de reflejos respiratorios. Según Joseph y col9, efectos adversos reconocidos de la anestesia regional en parturientas y no parturientas es hipotensión, arritmia y paro cardiaco.

Las arritmias cardíacas son causa importante de morbilidad - mortalidad durante el período perioperatorio, así como en pacientes críticos en unidad de cuidados intensivos. Las alteraciones del ritmo pueden ser bien toleradas por el corazón normal. Sin embargo, puede causar inestabilidad hemodinámica significativa en pacientes con un problema cardíaco congénito o adquirido ${ }^{1}$.

En el caso clínico presentado, la paciente no tenía ninguna alteración cardiaca al monitorizar y visualizar el electrocardiograma al inicio de la anestesia. Es importante recordar las alteraciones hormonales y hemodinámicos que ocurren durante el embarazo también pueden resultar en efectos pro-arrítmicos. El embarazo puede desencadenar el desarrollo de nuevas arritmias o agravar las que ya existen. Además, se demostró que la levobupivacaina y bupivacaina para la raquianestesia acortaron los intervalos QT de las pacientes. Los latidos atriales y ventriculares prematuros también son muy frecuentes ${ }^{4}$.

Podemos mencionar que la paciente presenta ansiedad y como describen Deniz y $\mathrm{col}^{4}$, Sato y $\mathrm{col}^{10}$ y Jang ${ }^{11}$, factores desencadenantes de bradicardia son: stress, emociones, dolor, ansiedad, miedo, fobia a la sangre, tos, micción y aumento de la actividad aferente del seno trigémino y carotideo. Shen y col ${ }^{12}$ describen las maniobras quirúrgicas como la externalización del útero, también pueden aumentar el tono vagal y causar arritmia.

Los agentes anestésicos pueden desencadenar actividad pro-arrítmica y antiarrítmica al inducir actividad eléctrica por medio de varios mecanismos. La bupivacaina es el medicamento más usado en anestesia obstétrica ${ }^{4}$. En nuestra paciente se utilizó bupivacaina pesada $10 \mathrm{mg}$. Existen reporte como menciona Leone y $\operatorname{col}^{5}$, que la disociación causada por la Bupivacaina es 10 veces más larga que la lidocaína y el bloqueo inducido por la bupivacaina puede acumularse, lo que resulta en una depresión cardiaca más marcada.

Por otro lado, la paciente presentó un aumento de la presión arterial dentro los 10 primeros minutos más la administración de atropina que estuvo elevado por 20 minutos más, posteriormente se observó descenso de la presión arterial que se mantuvo rangos de 100/60 mmhg a 103/62 mmhg. Mientras que, Ingersoll $\mathrm{y} \mathrm{col}^{13}$ indican que la dexmedetomidina tiene una respuesta bifásica aumento transitorio de la presión arterial seguido de una disminución sostenida la frecuencia cardiaca parece una similar combinación de una reducción mediada por baroreflexión de la frecuencia cardiaca que coincide con el aumento transitorios de la presión arterial, el tono simpático reducido mediado centralmente y aumento del tono vagal. Talke y $\mathrm{col}^{14}$ argumenta que el uso perioperatorio de $\alpha-2$ agonistas asociados con bradicardia, sedacion y sequedad en la boca y un paciente en su estudio presento pausa sinusal de 5 a 10 segundos durante la intubacion.

El objetivo de usar dexmedetomidina para prolongar el rescate analgésico y la disminución de la necesidad de analgésicos intravenosos, puede ser usado vía intravenosa, intranasal, intratecal, Xiong y col 15 describen la dexmedetomidina que puede prolongar la duración del bloqueo sensitivo y del bloqueo motor.

El recién nacido se registró APGAR de 8 y 9 al minuto y 5 minutos, Wang y col ${ }^{16}$ manifiestan que no existe efectos adversos en los recién nacidos y que la tasa de transferencia placentaria de la dexmedetomidina es de 0,68.

En nivel de dermatoma alcanzado en la paciente es de T5, según Owzuck y $\mathrm{col}^{17}$, el bloqueo simpático alto puede contribuir en inestabilidad hemodinámica que ocurren a causa de la anestesia regional y los agentes inotrópicos usados pueden ser causa de efectos proarrítmicos.

A los 10 minutos posterior a la administración de los medicamentos por vía intratecal, presento bradicardia de 39 latidos por minuto y se administró atropina $0,5 \mathrm{mg}$ y como menciona Afonso y $\mathrm{col}^{8}$, los efectos colaterales comunes de la dexmedetomidina son la bradicardia e hipotensión, los efectos temporales pueden ser controlados con atropina, efedrina e infusión de volumen.

Kalra y col 18 reporto en una paciente obstétrica la presencia de bradicardia seguida de un bloqueo Wenckebach atípico posterior a anestesia espinal.

En conclusión, la presencia de arritmias cardiacas puede 
deberse a varios factores, es trascendental la vigilancia y monitoreo continuo del electrocardiograma para reconocer y corregir de manera oportuna, en pacientes bajo anestesia regional, realizar lateralización uterina a la izquierda, prevención de hipotensión materna, administración de fluidos cocarga.

\section{Referencias bibliográficas}

1. Choudhury, M. Dexmedetomidine: The Anesthetic as an Antiarrythmic; Cardiol Pharmacol [Internert].2015 [Citado el 27 de junio de 2019];4(3). Disponible en: https://doi. org/10.4172/2329-6607.1000153

2. Hanci V, Ayoglu H, Yurtlu S, Yildirim N, Okyay RD, Erdogan G, Sayin E, Turan IO. An evaluation of $\mathrm{P}$ wave dispersion, QT, corrected QT and corrected QT dispersion intervals on the electrocardiograms of malnourished adults; Anaesth Intensive Care [Internet].2010 [Citado el 27 de junio de 2019];38(1), 122-127. Disponible en:https://journals.sagepub.com/doi/ pdf/10.1177/0310057X1003800120

3. Wang C, Liu S, Han C, Yu M, Hu Y, Liu C. Effect and placental transfer of dexmedetomidine during caesarean section under epidural anaesthesia; J Int Med Res [Internet]. 2017 [citado el 18 de mayo de 2019];45(3):964-972. Disponible en: doi:10.1177/0300060517698330

4. Deniz Y, Dilek Okyay, Volkan Hancı, Serhan Yurtlu, Hilal Ayoğlu, Işıl Özkoçak Turan, Efecto de la Levobupivacaína y la Bupivacaína en las Dispersiones de la Onda P, QT y QT Corregido (QTc) en Cesárea; Rev Bras Anesthesiol [Internet].2013 [Citado el 26 de junio de 2019];63(2):202 - 8. Disponible en: https://doi. org/10.1016/j.bjanes.2012.04.005

5. Leone S, Di Cianni S, Casati A, Fanelli G.Pharmacology, toxicology, and clinical use of new long acting local anesthetics, ropivacaine and levobupivacaine; Acta Biomed [Internet].2008 [Citado el 02 de julio de 2019];79(2), 92-105. Disponible en: http://www.embase.com/search/ results?subaction=viewrecord $\&$ from $=$ export $\&$ id =L352771924\%0Ahttp://www.actabiomedica.it/ data/2008/2_2008/leone.pdf

6. Carollo D, Nossaman B, Ramadhyani U. Dexmedetomidine: A review of clinical applications; Current Opinion in Anaesthesiology [Internet].2008 [Citado el 28 de junio de 2019]; 21(4), 457-461. Disponible en: https://doi. org/10.1097/ACO.0b013e328305e3ef
7. García Botero Andrés, Rodríguez Leonardo, Salazar Pérez Félix Arturo, Venegas Saavedra Alberto. Use of Dexmedetomidine in Total Intravenous Anesthesia (TIVA); Rev Colomb Anestesiol [Internet]. 2011 [Citado el 27 de junio de 2019]; 39(4): 514526. Disponible en:http://www.scielo.org.co/ scielo.php? script $=$ sci_arttext $\&$ pid $=$ S0 120 33472011000400005\&lng=en. http://dx.doi. org/10.5554/rca.v39i4.70

8. Afonso Joana, Reis Flávio. Dexmedetomidina: rol actual en anestesia y cuidados intensivos; Rev. Bras. Anestesiol. [Internet]. 2012 [Citado el 18 de junio de 2019]; 62(1):125133. Disponible en: http://www.scielo.br/ scielo.php? script $=$ sci_arttext $\&$ pid $=$ S0 034 70942012000100015\&lng=en. https://doi. org/10.1590/S0034-70942012000100015.

9. Joseph SE, Minehart RD. Third-degree heart block during spinal anesthesia for cesarean delivery; AA Case Rep [Internet].2014 [Citado el 27 de junio de 2019];3(1):3-5. Disponible en: https://doi.org/10.1213/XAA.0000000000000028

10. Sato S, Nakamori E, Kusumoto G, Shigematsu K, Yamaura K. Neurally Mediated Syncope During Cesarean Delivery: A Case Report; AA Case Rep [Internet]. 2017 [Citado el 02 de julio de 2019];8(5):96-99. Disponible en: https://doi. org/10.1213/XAA.0000000000000440

11. Jang YE, Do SH, Song IA.Vasovagal cardiac arrest during spinal anesthesia for Cesarean section; Korean J Anesthesiol [Internet].2013 [Citado el 27 de junio de 2019];64(1), 77-81. Disponible en: dx.doi.org/10.4097/ kjae.2014.64.1.77

12. Shen CL, Ho YY, Hung YC, Chen PL. Arrhythmias during spinal anesthesia for Cesarean section; Can J Anaesth [Internet].2000 [Citado el 27 de junio de 2019];47(5):393-397. Disponible en: doi:10.1007/BF03018966

13. Ingersoll-Weng Esperanza, Manecke Gerard, Thistlethwaite Patricia. Dexmedetomidine and Cardiac Arrest; Anesthesiology [Internet].2004
[Citado el 20 de febrero de 2020];100(3), 738-39. Disponible en: https://doi.org/10.1097/00000542200403000-00037

14. Talke Pekka, Chen Richard, Thomas Brian, Aggarwall Anil, Gottlieb Alexandru, Thorborg Per, Heard Stephen, Cheung Albert, Son Stanley, Kallio Antero. The hemodynamic and adrenergic effects of perioperative dexmedetomidine infusion after vascular surgery; Anesthesia and Analgesia [Internet].2000 [Citado el 23 de febrero de 2020]; 90(4), 834-39. Disponible en: https:// doi.org/10.1097/00000539-200004000-00011

15. Xiong Yaqin, Ma Yushan, Wu Lan, Luo Dong. Case Report Intravenous dexmedetomidine as an adjuvant in anesthetic management of a paturient for cesarean section with uncontrolled hyperthyroidism; Int J Clin Exp Med [Internet].2016 [Citado el 27 de junio de 2019];9(8),16913-17. Disponible en:https://pdfs. semanticscholar.org/b39e/6baea5e61554ee92e410 489d53d53613b28d.pdf

16. Wang Changsheng, Liu Shijiang, Han Chuanbao, Yu Min, Hu Youli, Liu Cunming. Effect and placental transfer of dexmedetomidine during caesarean section under epidural anaesthesia; J Int Med Res [Internet].2017 [Citado el 18 de mayo de 2019];45(3):964-972. Disponible en: https://doi. org/10.1177/0300060517698330

17. Owczuk Radoslaw, Sawicka Wioletta, Wujtewicz, Magdalena Anna, Kawecka Aleksandra, Lasek Jerzy, Wujtewicz Maria. Influence of spinal anesthesia on corrected QT interval; Regional Anesthesia and Pain Medicine [Internet].2005 [Citado el 03 de junio de 2019];30(6),548-52. Disponible en: https://doi. org/10.1016/j.rapm.2005.06.005

18. Kalra Seema, Hayaran Nitin. Case Report Arrhythmias following spinal anesthesia for cesarean delivery - Is Wenckebach common? J Anaesthesiol Clin Pharmacol [Internet].2011[Citado27 de junio de 2019];27(4),541-44. Disponible en: https://doi. org/10.4103/0970-9185.86604 